\title{
Tissue Engineering with Adult Stem Cell Sheets
}

\section{Ram V. Devireddy*}

Department of Mechanical Engineering, Louisiana State University, Baton Rouge, USA

\section{Adult Stem Cell Tissue Engineering}

The potential of stem cells to differentiate along multi-lineage pathways, and produce more specialized cells of the body provides: i) a tool to study the early human embryonic development and, ii) to develop novel strategies of organ regeneration. The central nervous system yields neuronal stem cells that can differentiate and produce not only neuronal cells but also other tissues, including blood and muscle. Stem cells from the liver can be converted, in vitro, into insulin-secreting pancreatic cells. Of the many different types of adult stem cells, the most widely known and best studied are the bone marrow stem cells. These bone marrow derived mesenchymal stem cells have been successfully differentiated into various cell lineages including neuronal, cartilage, bone, tendon, muscle, and fat lineages. Bone marrow mesenchymal stem cells transplanted into mice can go on to differentiate into astrocytes, neurons, and oligodendrocytes, bone or other lineages. Although advances in cytokine technology make it possible to mobilize stem cells from the marrow into the peripheral circulation for easier access, new research has demonstrated that adult stem cells similar to the bone marrow mesenchymal stem cells are plentiful in human fat available via liposuction harvest, which in contrast, routinely delivers liter volumes of tissue. The adipose tissue derived progenitor cells exhibit multi-lineage potential and express biochemical markers and feature in vitro consistent with bone, cartilage, epithelial, fat, liver, muscle, and nerve cells. Moreover, human adipose-derived stem cells have been used successfully to repair a damaged region in a skull. In order to harness the potential therapeutic benefits of Adipose-derived Stem Cells (ASCs) for tissue engineering strategies and cell-based therapies, we will require development of simple protocols for the development of uni- and multi-dimensional tissue-like products.

\section{Clinical Problem}

Orthopedic injuries and osteoporosis are common medical problems. Musculoskeletal defects restricted the lives of over 36 million Americans in 1995 and the numbers have only increased during the intervening years. Many of these disorders require surgery to accelerate or improve bone repair. The "perfect" graft material for a spinal fusion would provide the following properties: Mechanical support: Material stabilizes the spine/surgical site during the recovery period; Osteoconductive: Material facilitates the ingrowth and integration of adjacent bone upon itself; Osteoinductive: Material recruits and stimulates the formation and growth of bone from cells that may not naturally do so and; Osteogenic: Material contains cells that themselves are capable of forming new bone. While there are alternative materials available, all face a common limitation; none display osteogenic capability. Allograft bone from cadavers can be sterilized, stored, and used in the operating room as needed; however, allografts can cause inflammation, elicit an immune response, and have been an infectious source in a limited number of cases. Ceramic materials, such as hydroxyapatite and tricalcium phosphate (HA/TCP), are osteoconductive and will promote new bone formation. However, they lack osteogenic and osteoinductive properties, limiting their utility.

\section{Cell Sheet Engineering}

There has been considerable progress in developing cellularized scaffolds to regenerate various tissues including bone, cartilage, blood vessel, nerve, liver, myocardium and many other tissues. However, most of the conventional scaffolds do not allow sufficient cell migration to establish adequate cell-extracellular matrix (ECM) and cell-cell interaction. To overcome some of the drawbacks associated with traditional scaffold based tissue engineering, cell sheet engineering has been developed as an alternative approach for tissue engineering. Adapting these earlier cell sheet engineering technologies we have developed a modified MC-collagen hydrogel method to create cell sheets, specifically tailored to work with ASCs.

\section{Fabrication of ASC sheets}

Our ASC culture experiments were performed by adding $\sim 200,000$ cells $/ \mathrm{ml}$ in ASC cell culture media on the $14 \%$ MC-water- $0.5 \mathrm{x}$ PBS hydrogel system (mol. wt. $=15,000)$. Initially the ASCs were completely unreceptive to the hydrogel surface and generated embryoid-like structures, as opposed to the cell sheets that we were hoping for (Figure 1A). Fortunately, the addition (evenly spread) of $200 \mu \mathrm{l} \mathrm{of} 2 \mathrm{mg} / \mathrm{ml}$ bovine collagen type-I ( $\mathrm{pH}$ adjusted to 7.5 ) over the MC coated surface at $37^{\circ} \mathrm{C}$ (Figure $1 \mathrm{~B}$ ), significantly improved the stem cell adhesion and proliferation on the hydrogel system (Figure 1C). Thus, we were able to grow ASC sheets on a thermally reversible hydrogel system. Upon confluence, a continuous monolayer ASC sheet was formed on the surface of the MC Hydrogel system (Figure 1C). When the grown cell sheet was removed from the incubator and exposed to room temperature $\left(\sim 30^{\circ} \mathrm{C}\right)$, it spontaneously and gradually detached from the surface of the thermo-responsive hydrogel (without the use of any detachment enzymes, like trypsin). The ASC cell sheet started detaching from its edge at $\sim 1$ min after being exposed to room temperature. Detachment of the entire cell sheet was completed within 2 to 3 mins. Since, these cells were never exposed to trypsin and other detachment enzymes, it is to be expected that the binding integrins are not compromised in the cell culture/growth process.

Further development of potential therapeutic treatments using these newly developed MC hydrogel systems requires a better understanding of the underlying mechanisms that regulate ASCs differentiation in a $2 \mathrm{D}$ cell sheet environment, as opposed to the traditional development mode of "attached" to a Tissue Culture Polystyrene (TCPS) surface.

*Corresponding author: Ram V. Devireddy, Department of Mechanical Engineering, Louisiana State University, Baton Rouge, LA 70803, USA, Tel: 1(225)589-5891; Fax: 1(225)578-5924; E-mail: devireddy@me.Isu.edu

Received October 17, 2012; Accepted October 18, 2012; Published October 22 2012

Citation: Devireddy RV (2012) Tissue Engineering with Adult Stem Cell Sheets. J Bioengineer \& Biomedical Sci 2:e106. doi:10.4172/2155-9538.1000e106

Copyright: @ 2012 Devireddy RV. This is an open-access article distributed under the terms of the Creative Commons Attribution License, which permits unrestricted use, distribution, and reproduction in any medium, provided the original author and source are credited. 

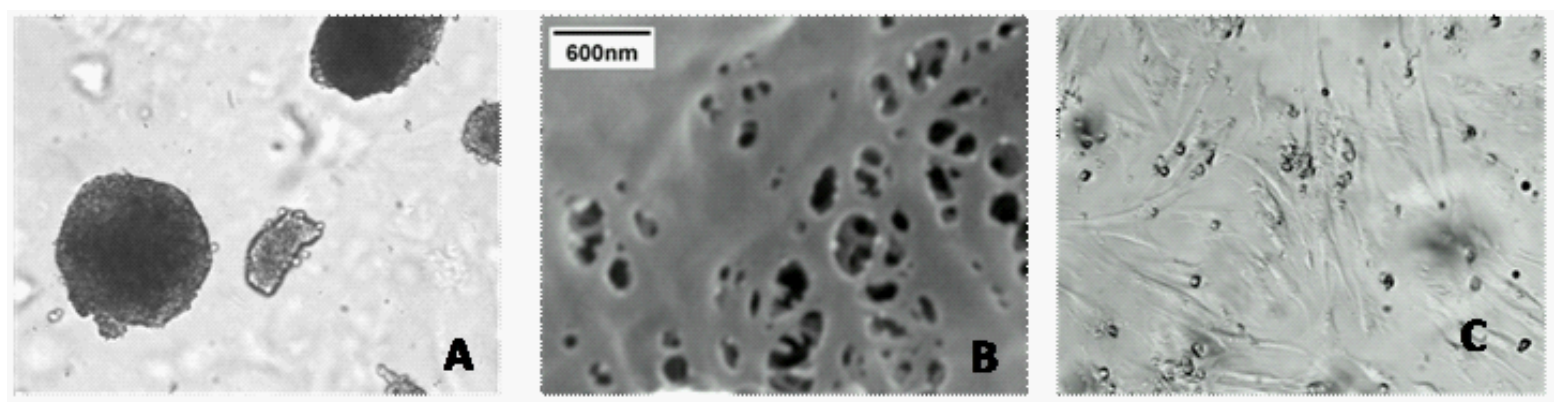

Figure 1: (A) ASCs grown on MC hydrogel system. (B) SEM image of the collagen network formed onto the MC coated TCPS dish. (C) ASCs grown on MC hydrogel system coated with collagen. 